\title{
The Research of Platform for Bridge Visual Inspection Based on Quadrotor Aircraft
}

\author{
Zhensong $\mathrm{Ni}^{1 \text {, a }}$, Shuri Cai ${ }^{2, \mathrm{~b}}$ and Jing Liu ${ }^{2, \mathrm{C}}$
}

\author{
${ }^{1}$ The school of Electronics and Information Engineering, Fuqing branch of fujian normal university of \\ electronic and information engineering, Fujian, China \\ ${ }^{2}$ Research Institute of Highway, Ministry of Transport, China. \\ A460532802@qq.com, br.cai@rioh.cn, ${ }^{\text {c5} 568251076 @ q q . c o m ~}$
}

Keywords: Vertical Take-Off and Landing; unmanned aerial vehicles;bridge inspection vehicle;.

\begin{abstract}
Table view examination is one of the important means and basic steps of the bridge structure. It founds up less than $0.2 \mathrm{~mm}$ small cracks in up to hundreds of meters or tens of kilometers long buildings. It must be close to the observed. However, the modern bridge structure is diverse, the surrounding environment is complex, and the appearance of detection is difficult to achieve. In this paper, the problem of bridge appearance detection is discussed, and the application prospect of the four rotors unmanned aerial vehicle is discussed. At the same time, this paper also discuss about how to improve the four rotor unmanned aerial vehicle to be applied to the preliminary exploration of the appearance of the bridge structure, and preliminary indoor environmental testing, testing its feasibility. As it can be seen from the test results, the four rotors unmanned aerial vehicle can be applied in the appearance of the bridge detection. Finally, the paper analyzes and summarizes the challenges of the four rotors unmanned aerial vehicle in the bridge appearance detection.
\end{abstract}

\section{Introduction}

By the year of 2009, China's total mileage of highway has reached $3860800 \mathrm{~km}$, and the number of highway bridges has amounted to 621 900, making China the country that owns the most bridges according to the latest statistical bulletin of the development of highway and waterway transportation from the China's Ministry of Transport. Bridges are important highway infrastructures and the construction of them costs much. Their qualities have a direct impact on the safe and quick flowing traffic. Under the circumstance of highway networks, once bridges collapse or are destroyed by accident, it will cause huge economic lose and other damages, exerting an unbearable social effect on modern transportation system, and the situation turns out to be worse especially for large bridges. Thus a higher level of infrastructure structure for important highway and safety of traffic is required. In order to avoid the potential safety problems in bridge structure, Specification for Highway Bridge Maintenance, a China's transportation industry standard, has developed detailed approaches for Bridge maintenance and management, in which visual inspection of bridge is one of the important means and basic steps to identify problems. However, the diversity of modern bridge structures and the complex surroundings make it difficult to implement visual inspection. The reasons are as follows: (1) Bridges are buildings across obstacles, such as rivers, valleys, roads, railways, etc; ${ }^{(2)}$ Clear height and clear span of bridges vary widely. The former can range from one meter to hundreds of meters, and the latter from several meters to thousands of meters; ${ }^{(3)}$ The objects of bridge visual inspection include not only bridge floors but also piers and the bottoms and sides of girders; ${ }^{(4)}$ In order to discover the tiny gap less than 0.2 millimeter in a huge building as long as hundreds of meters or dozens of kilometers (for example, Hangzhou Bay Bridge), close approach to the observed object is necessary.

However, the most difficult is that the test objects are usually difficult to reach. With advances in science and technology, bridge inspection vehicles can help bridge inspectors to have a more detailed examination of the bottoms of bridge girders. But there are disadvantages for using bridge inspection vehicles. ${ }^{(1)}$ Renting bridge inspection vehicles costs much, about 10,000 to 20,000 Yuan or so per day 
on average. (It will cost more than 5 million Yuan to purchase one bridge inspection vehicle.) ${ }^{(2)}$ For the traffic safety, traffic control measures must be taken since the bridge inspection vehicle is relatively large, occupying one traffic lane and a half. ${ }^{(3)}$ Inspecting efficiency is low, for it takes time to readjust the inspection vehicle for the inspection of every span, as shown in Figure $1 .{ }^{(4)}$ Some places, such as towers, arch bridges across valleys or rivers and so on, are inaccessible for the inspection vehicle, and there are many blind spots for inspection. ${ }^{(5)}$ It will constitute a danger to personal safety of users under certain circumstances like traffic accidents, extreme weather events of thunderstorm or typhoon etc.

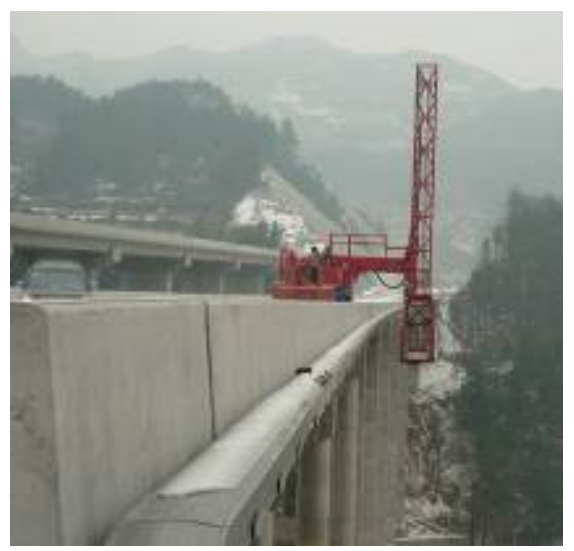

Figure 1 Bridge Inspection Vehicle

In addition, the 5.12 Wenchuan earthquake in Sichuan Province has brought a lot of reflection on China's transportation. The highway traffic was completely cut off and bridge inspection vehicles were not available in the earthquake zones at that time. However, the government was in dire need of being aware of the extent of the damage to the local highways and bridges in order to work out a scientific strategy to deliver relief supplies. Under this circumstance, the measures for bridge visual inspection were back to the primitive state of hiking and using telescopes, thus the reliability totally depends on inspectors' experiences and perception.

Although Wenchuan earthquake brought a catastrophe to Sichuan Province, the relief action by air from the government and the application of unmanned aerial vehicles (UAV) has inspired experts. If UAVs can be applied to bridge visual inspection with more sophisticated technologies in this field, these UAVs can be available both for daily use and in emergency after a disaster, avoiding bridge inspection vehicles' limitation. There is no doubt that UAVs will become a more ideal inspection platform than bridge inspection vehicle.

\section{Design model of four rotor unmanned aerial vehicle for bridge application}

The current researches on quad-rotor UAVs are using the simple mechanical prototype of a quad-rotor UAV shown in Figure 2 to test, model and simulate. Taking long-range videos and photos through quad-rotor UAVs has been put into commercial use.And for bridge appearance inspection, the four rotor unmanned aerial vehicle (UAV) must have docked on the structure surface, because for the cracks of concrete surface observation, the bridge maintenance and management personnel are more concerned about is crack width and crack width of observation, must meet the following conditions: Photo shoot has sufficient resolution, at least to identify cracks $0.1 \mathrm{~mm}$ or more, in the four rotor unmanned flight load capacity is insufficient, the general can only use ordinary camera and camera, which must be close to the surface of the building. To be closer to the surface of the bridge structure,Unmanned aerial vehicles shown in Figure 2 must be improved. It can avoid leading to unmanned aerial vehicles damaged or out of control and crashed due to safety problems.

At the same time, to make the four rotor unmanned aerial vehicle (UAV) can be applied to the detection of the bridge appearance,It is considered in this paper, through two kinds of means to achieve common, one Quad rotor unmanned aerial vehicles can paste bridge surface sliding; the second is to increase the scale, unmanned aerial vehicles in the structure of the sliding surface, scale 
next to the surface structure, so that the detection of bridge cracks in the bottom, close to the surface of a building can be according with scales of crack images for estimating bridge crack width.

Figure 3 shows the physical structure model of the four rotor unmanned aerial vehicle, which is improved for the application of bridge appearance detection.Compared with figure 2, figure 3 physical model is to increase the content of three parts, one is from the center to four wing connecting rod of the extension of a certain degree of, and then use the annular elastic materials connected, the circular retainer must be more than four rotor wing pulp, to ensure that the rotor will not encounter structures affect flight, as shown in Figure 3 (left). The other is the four connecting rod places to increase the vertical column, its height should be more than four rotor wing height, to ensure that unmanned aerial vehicles close to the surface of the building will not damage the aircraft, as shown on the right side of the Figure 3; The three is to add a scale in the diagonal of the four columns, as shown in Figure 4 on the right.

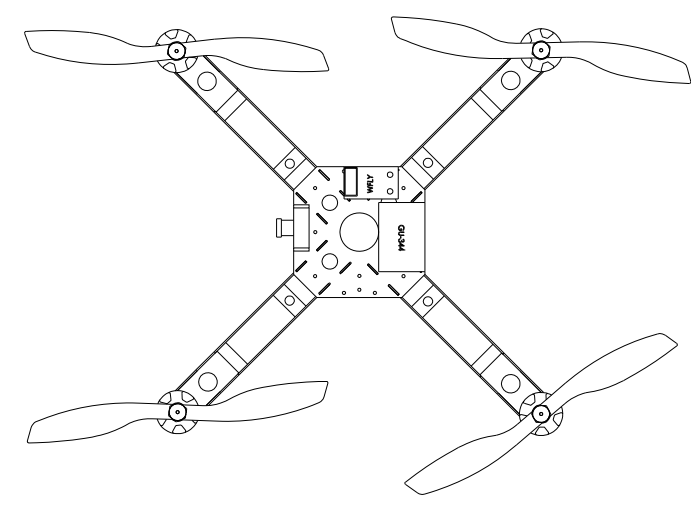

Figure 2 A simple mechanical prototype of a quad-rotor UAV
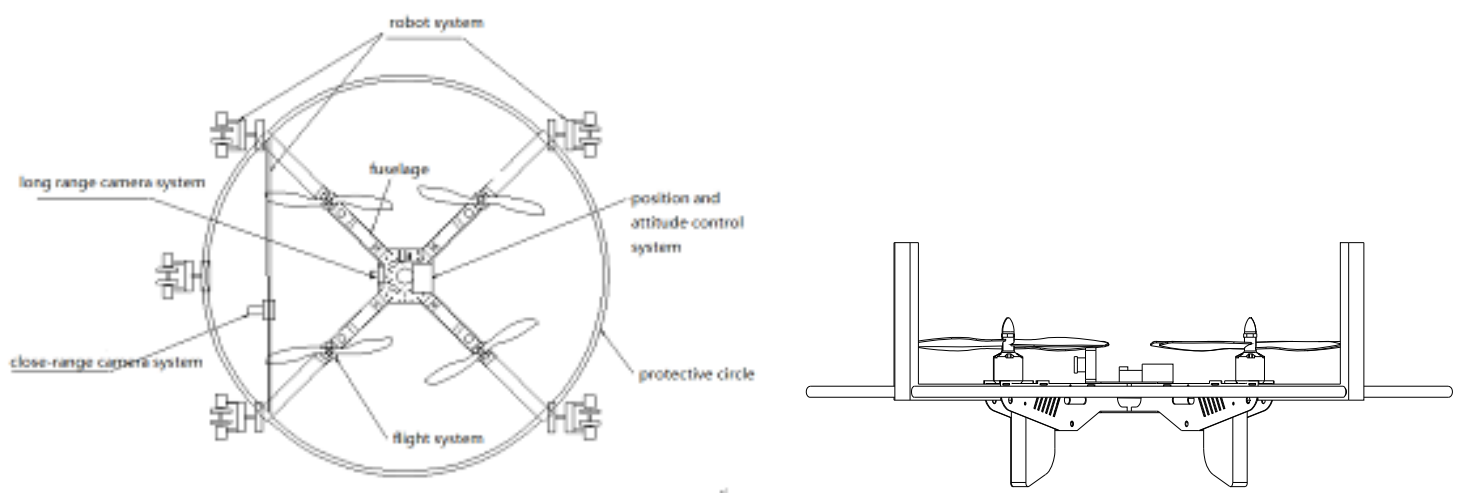

Figure 3 The mechanical top and side view of a quad-rotor UAV with protective accessories

\section{Four rotor unmanned aerial vehicle inspection test}

A upgraded model from the model of GAUI (a Taiwanese brand) 330 brought by the research group is shown in Figure 4.Taking into account the main testing site of the main appearance, here to do two tests, one unmanned aerial vehicle adsorption at the bottom of the structure of the test, the other is Figure 6 demonstrates the feasibility test of the upgraded quad-rotor UAV carried out by the research group. After it adheres to the bottom of the bridge girder, the UAV can use its close-range camera system to take photos. The control is very inconvenient because it is operated by man. But it was found through preliminary experiments that the control of UAV changes obviously due to the extra protective accessories and auxiliary equipment, thus it is necessary to re-model and re-simulate according to the new model.

Top stop test. Figure 4 shows an improved aircraft structure for the top docking program: 


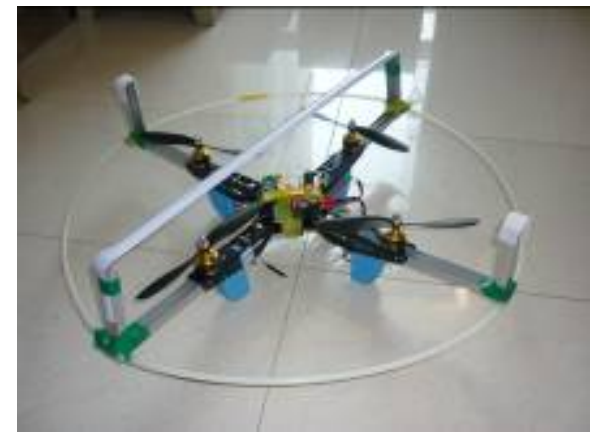

Fig. 4 the structure of the aircraft at the top of the program

Figure 4 shows in the outside of the four rotor installed four columns, and the height of the pole to much higher than the rotor height, so that you can rely solely on the four rotor lift an aircraft is firmly anchored in sunshine surface structure, like it is adsorbed in the surface, two of them were diagonally at the top of the upright post tied a ruler (as a measure of crack width).
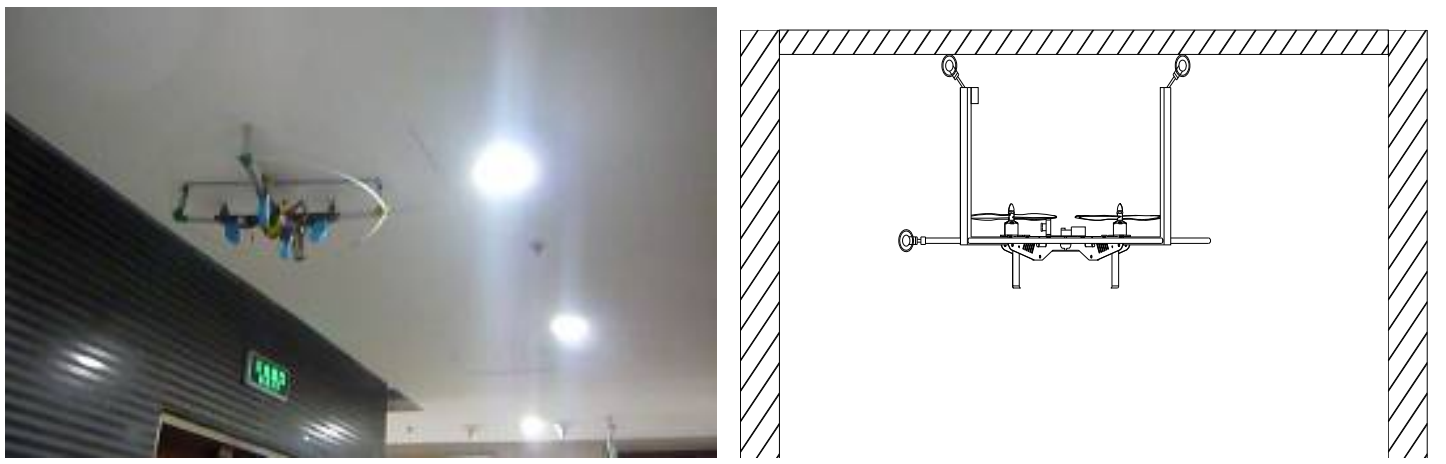

Fig. 5 The bottom test and physical model of the adsorption structure

Fig. 5 shows the aircraft adsorption test at the bottom of the structure , according to the test, in the case of sufficient power, the aircraft docking effect is very stable, in the central part of the aircraft is install a wireless camera,Fig. 6 shows the aircraft adsorption at the bottom of the structures, A crack screen shot by a wireless camera, The smallest unit of scale is a millimeter, The scale of each scale is about 0.1 0.2 $\mathrm{mm}$. Therefore, Although the resolution of the wireless camera is relatively low. Only $360 * 240$ resolution, But if you can achieve the test as shown in figure 5, Close to the surface of the building,Through the image measure the width of the crack is enough.

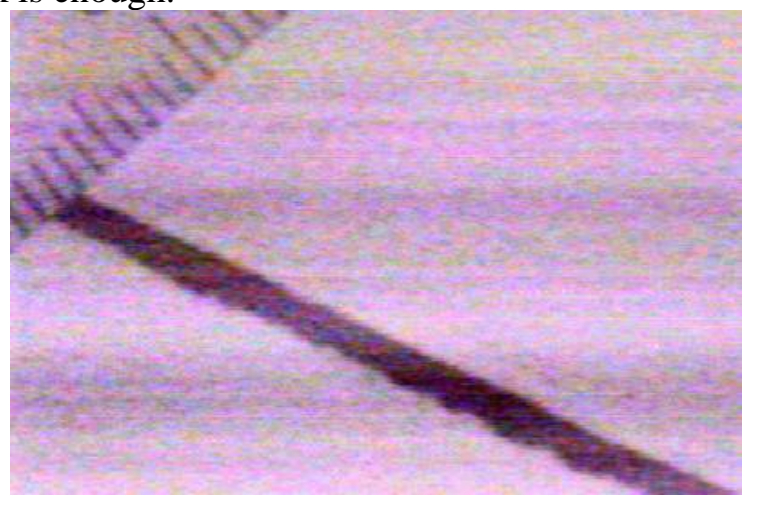

Fig 6. the crack detection camera video capture of the bottom test of the adsorption structure

Side stop test.Fig 7 shows the form of an improved aircraft structure for side docking.In the test, the side of the parking gesture to maintain about 30 seconds, the need shooting time for crack detection has been very full.Two experimental tests of this section show that the detection of cracks on the surface of the four rotor aircraft which is close to the surface of the bridge is completely feasible. 


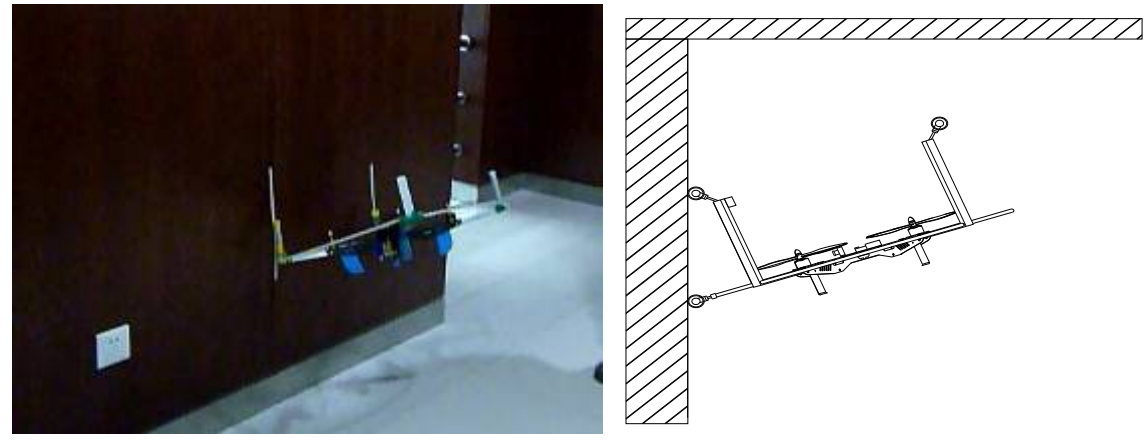

Fig. 7 side parking test and physical view of aircraft

\section{Summary}

If you follow the "checklist" your paper will conform to the requirements of the publisher and facilitate a problem-free publication process. Its $\mathrm{R} \& \mathrm{D}$ process not only solve the problems of their own existence,but also it has actively promoted and promoted the development of many other related technologies.In this paper, the research and application of the four rotor unmanned aerial vehicle is Preliminary exploration in Bridge appearance detection.And a certain transformation for the four rotor unmanned aerial vehicle, So that it can be applied to the bridge environment.Indoor environmental testing shows:Improvement measures are indeed feasible.

\section{Acknowledgements}

This work was financially supported by the Fujian Office of Education Foundation (JA15573 ).

\section{References}

[1] Phillip McKerrow. Modeling the Draganflyer four-rotor helicopter[C], Proceedings of the 2004 IEEE International Conference on Robotics \& Automation New Orieans, LA April 2004.

[2] Steven L. Waslander, Gabriel M. Hoffmann, Jung Soon Jang, Claire J. Tomlin. Multi-Agent Quadrotor Testbed Control Design: Integral Sliding Mode vs. Reinforcement Learning[C], 2005 IEEE/RSJ International Conference 2-6 Aug. 2005.

[3] Altug E., Ostrowski J.P., Taylor C.J. Quadrotor control using dual camera visual feedback[C], Proceedings of IEEE International Conference on Robotics and Automation, Taipei, Taiwan, 2003, PP.4294-4299.

[4] Bouabdallah S. Design and Control of Quadrotors with Application to Autonomous Flying[D], Lausanne, EPFL 2006.12.

[5] V. Mistler, A. Benallegue, N.K. M’Sirdi. Exact Linearization and Noninteraeting Control of a 4 Rotors Helicopter via Dynamic Feedback[C].IEEE 10th IEEE International WorkshoP on Robot-Human Interactive Communication Bordeaux and Paris, 2001. 